\title{
Inhibitory Mechanism of Salidroside on Tyrosinase
}

\author{
Yaxin Zhu ${ }^{1}$, Chaoyin Chen ${ }^{1, *}$, Shenglan Zhao ${ }^{2, *}$, Jiong Yang ${ }^{1}$, Hao Song ${ }^{1}$, Feng Ge ${ }^{1}$, Diqiu Liu ${ }^{1}$ \\ ${ }^{1}$ Faculty of life science, Kunming University of Science and Technology, Kunming, Yunnan, China \\ ${ }^{2}$ Yunnan University of Traditional Chinese Medicine, Kunming, Yunnan, China \\ *Corresponding author: chaoyinchen@163.com; zhaoshenglan@163.com
}

Received September 03, 2014; Revised September 19, 2014; Accepted September 26, 2014

\begin{abstract}
Tyrosinase is a key rate-limiting enzyme in melanin synthesis which can be blocked to reduce melasma and freckles by inhibiting the activity of tyrosinase. Salidroside showed certain inhibitory effects on tyrosinase by enzyme kinetics with inhibition of 33.04\%. Besides it was also a competitive inhibitor of tyrosinase which served as a substrate analogue to combine with enzyme to produce quinones with stronger light absorption. Furthermore, docking results implied that inhibitory mechanisms might be attributed to the weak interactions between salidroside and tyrosinase such as hydrogen bonds and van del waals. Salidroside is a main kind of plant flavonoids from Rhodiola rosea, walnut and so on. Studying inhibitory mechanism of salidroside on tyrosinase is not only helpful for developing anti-freckle products in which salidroside is one of the most important components, but also beneficial for making better use of plant flavonoids to seek for a new generation of natural skin care cosmetics.
\end{abstract}

Keywords: tyrosinase, salidroside, inhibitor, mechanism, flavonoid

Cite This Article: Yaxin Zhu, Chaoyin Chen, Shenglan Zhao, Jiong Yang, Hao Song, Feng Ge, and Diqiu Liu, "Inhibitory Mechanism of Salidroside on Tyrosinase." Journal of Food and Nutrition Research, vol. 2, no. 10 (2014): 698-703. doi: 10.12691/jfnr-2-10-8.

\section{Introduction}

Tyrosinase (EC 1.14.18.1) also known as polyphenol oxidase (PPO) is a multifunctional copper-containing oxidase which is widely distributed in plants, animals and microorganisms [1]. Melanin, the major pigment in skin, plays an important role in protecting the skin against ultraviolet rays owing to its ability to absorb ultraviolet light and scavenge oxidative free radicals [2,3,4,5]. However, excessive melanin formation and accumulation of melanin can cause skin hyperpigmentation such as melasma and freckles [6]. The biosynthetic pathway of melanin includes following steps: Tyrosine is gradually oxidized to dopamine and dopamine quinone by tyrosinase. Then after rearrangement and a series metabolic process, they are aggregated into 5,6dihydroxyindole and indole quinone. Eventually, melanin is formed $[7,8]$. Throughout the course of producing melanin, tyrosinase which is the main rate-limiting enzyme in formation process of melanin is both served as hydroxylase of tyrosine and dopa oxidase and oxidase of 5,6-dihydroxyindole $[9,10]$. The inhibition of melanin in skin mainly contains intracellular and extracellular inhibition of melanocyte. The inhibition of tyrosinase belongs to intracellular inhibition. According to different mechanisms of inhibition, the inhibition of tyrosinase can be divided into destructive and nondestructive Inhibition. The former is achieved by inhibiting tyrosinase synthesis or replacing substrates of tyrosinase to block the formation of melanin, while the latter is realized by modifying and transforming the tyrosinase [11]. The mechanism related to lots of anti-freckle products is generally connected with the ability of products to decrease melanin synthesis by inhibiting activity of tyrosinase, formation of melanin, and transference of melanosomes from melanocytes to keratinocytes [12,13]. At present, there are some compounds that also can inhibit the activity of tyrosinase, such as kojic acid and arbutin [14,15]. Nevertheless, the compounds have side effects, particularly low clinical efficiency and skin toxicity $[16,17]$. Therefore, it is necessary to develop new natural anti-freckle products among which phenolic and polyphenolic compounds of plant origin are likely to be another effective and safe skin care cosmetics $[18,19,20]$.

Salidroside, a compound with the chemical structure of phenol glycosides, is an important kind of flavonoids extracted from the root of Rhodiola rosea [21], existed in seed coat of walnut and so on [22,23]. Besides roles in anti-inflammation [24], anti-oxidation [25], scavenging free radical [20], salidroside also can reduce ischemiamediated myocardial damage [26], moreover, it has been shown that salidroside has ability to inhibit the activity of tyrosinase which is the main rate-limiting enzyme in the process of melanin synthesis [20]. So far there have been some cosmetics added to extracts in which salidroside is the main ingredient, however, until now no researches have been carried to explore the inhibitory mechanism of salidroside on tyrosinase. Thus the aim of this paper is to study the inhibitory mechanism of salidroside on tyrosinase in order to make better use of salidroside or extracts in which salidroside is the main ingredient to develop new anti-freckle products.

\section{Materials and Methods}




\subsection{Material and Chemicals}

Salidroside used in this study was purchased from Xi'an Kailai biological engineering Co. Ltd. (China). L3,4-Dihydroxyphenylalanine (L-DOPA) was acquired from Xi'an Huilin Biological technology Co. Ltd. (China). $\mathrm{CuSo}_{4}$ was from Tianjin Fengchuan Chemical Reagent Co. Ltd. (China). Dimethylsulfoxide (DMSO) was provided by MP Biomedicals LLC (U.S.). Mushroom tyrosinase (EC 1.14.18.1) was the product of the Worthington Biochemical Corporation (U.S.). The specific activity of the enzyme was $845 \mathrm{U} / \mathrm{mg}$. All other reagents were of analytical grade. Ultrapure water was used for the whole process.

\subsection{Tyrosinase Activity Assay}

The tyrosinase activity assay was performed as previously described with some modifications [27,28,29]. The reaction media $(3 \mathrm{ml})$ for tyrosinase activity assay contained $0.5 \mathrm{mM}$ L-DOPA in $50 \mathrm{mM}$ sodium phosphate buffer ( $\mathrm{pH}$ 6.8), $0.1 \mathrm{ml}$ of different concentrations of inhibitor that was dissolved in DMSO of which the final concentration in the test solution was $3.3 \%$, and $0.1 \mathrm{ml}$ of mushroom tyrosinase of which the eventual concentration was $6.67 \mu \mathrm{g} / \mathrm{ml}$. Throughout the process, firstly $0.1 \mathrm{ml}$ of different concentrations of inhibitor dissolved in DMSO solution was added into the test tube. Then $2.8 \mathrm{ml} \mathrm{L-}$ DOPA in $50 \mathrm{mM}$ sodium phosphate buffer was also put into the test tube, and at the same time the temperature was controlled at $30^{\circ} \mathrm{C}$. After incubation for $10 \mathrm{~min}, 0.1$ $\mathrm{ml}$ of the aqueous solution of mushroom tyrosinase was added, immediately the solution was monitored for $1 \mathrm{~min}$ for the formation of dopachrome by measuring the linear increase in absorbance at $475 \mathrm{~nm}$ accompanying the oxidation of the substrates with a molar absorption coefficient of $3700\left(\mathrm{M}^{-1} \cdot \mathrm{cm}^{-1}\right)$ by ultraviolet spectrophotometer. The inhibition of tyrosinase activity was calculated as follows:

$$
\text { Inhibition(\%) }=\left(1-\frac{A_{3}-A_{4}}{A_{1}-A_{2}}\right) \times 100 \%
$$

Where $A_{1}$ was the absorbance at $475 \mathrm{~nm}$ with tyrosinase and substrate, $A_{2}$ was only with tyrosinase, $A_{3}$ was the absorbance at $475 \mathrm{~nm}$ with tyrosinase, substrate and inhibitor, $\mathrm{A}_{4}$ was also with tyrosinase and inhibitor finally.

Keep concentration of substrate unchanged and measure the catalytic activity of mushroom tyrosinase at different enzyme concentrations and different concentrations of inhibitor in order to research that whether the inhibition of salidroside on tyrosinase is reversible or not. Then remain unchanged enzyme concentration and study the catalytic activity of mushroom tyrosinase at different concentrations of substrate and different concentrations of inhibitor so as to determine the inhibitory type of salidroside on tyrosinase by lineweaverburk plots [29].

\subsection{UV-VIS Spectra}

The ultraviolet-visible spectroscopy was used to study the chelation of salidroside and copper ions in the active center of tyrosinase. Prepare three test tubes which were signed with $1,2,3$, and respectively added to $2.8 \mathrm{ml}$ ultrapure water and $0.1 \mathrm{ml}$ salidroside that was dissolved in DMSO. Then the first test tube was added to $0.1 \mathrm{~mL}$ tyrosinase which was dissolved in sodium phosphate buffer, and the second was put in $0.1 \mathrm{~mL} \mathrm{CuSO}_{4}$ solution, meanwhile the third was added to $0.1 \mathrm{~mL}$ sodium phosphate buffer. Keep temperature of $30^{\circ} \mathrm{C}$ for $10 \mathrm{~min}$. Finally, Scan ultraviolet-visible spectrum of each mixture respectively in the wavelength of 200-600 $\mathrm{nm}$ in the reaction of $0,10,20,30$, and $40 \mathrm{~min}[30,31]$.

\subsection{Model Building and Molecular Docking}

Molecular docking was performed to investigate the binding mode of salidroside and tyrosinase by means of Autodock Vina version 1.1.2 [32]. The three-dimensional coordinate of the copper-bound tyrosinase (PDB ID: 2ZWG) was downloaded from Protein Data Bank (http://www.rcsb.org/pdb/home/home.do) and was used as the input structure for docking calculations because of the similarity between salidroside and 3,4dihydroxyphenylalanine. The AutoDock Tools version 1.5.6 package (http://mgltools.scripps.edu) was employed to generate the docking input files. Ligand structure was prepared for docking by merging non-polar hydrogen atoms and defining rotatable bonds. The search grid of tyrosinase was identified as center_x: -14.594, center_y: 16.832, and center_z: 22.285 with dimensions size_x: 15, size_y: 15, and size_z: 15 . In order to increase the docking accuracy, the value of exhaustiveness was set to 20. For Vina docking, the default parameters were used as described in the Autodock Vina manual unless otherwise specified. The top ranked pose as judged by the Vina docking score was subject to visually analyze using PyMOL software (http://www.pymol.org/).

\section{Results and Discussion}

\subsection{Effects of Salidroside on the Activity of Tyrosinase}

Through studying the reaction media $(3 \mathrm{ml})$ for tyrosinase activity assay that contained $2.8 \mathrm{ml}$ of $0.5 \mathrm{mM}$ L-DOPA in $50 \mathrm{mM}$ sodium phosphate buffer ( $\mathrm{pH}$ 6.8), 0.1 $\mathrm{ml}$ of different concentrations of inhibitor and $0.1 \mathrm{ml}$ of $6.67 \mu \mathrm{g} / \mathrm{ml}$ mushroom tyrosinase, the effects on the oxidation of L-DOPA of salidroside at different concentrations by mushroom tyrosinase were tested. Salidroside showed inhibitory effects on tyrosinase activity (Figure 1). What's more, when the concentration of salidroside was up to $0.1 \mathrm{mM}$, the highest inhibition rate of tyrosinase by salidroside reached 33.04\%. Whereas, when the concentration of salidroside was over $0.1 \mathrm{mM}$, with the increasing concentration of inhibitor, the inhibition of tyrosinase by salidroside was reduced. According to the following study, salidroside was served as substrate analogue to react with tyrosinase. When concentrations of inhibitor became too high, the inhibitors competed with each other to combine with the enzyme, which might lead to the change of spatial structure of enzyme. However, the change might be helpful for the combination between substrate and enzyme, so the inhibition rate of salidroside on tyrosinase was reduced. In a word, the result suggested that salidroside had ability to inhibit the activity of tyrosinase, and also could reduce melasma and freckles to some extent. 


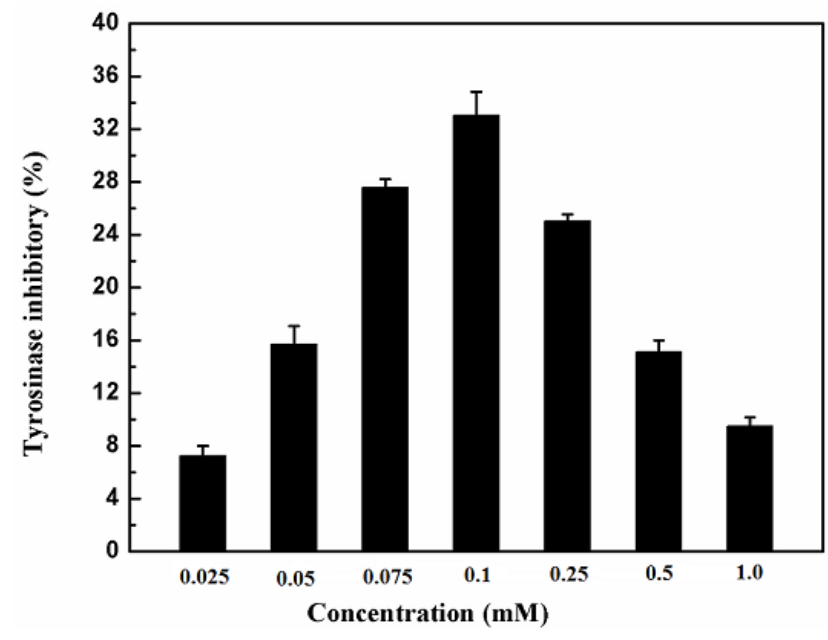

Figure 1. Inhibition of tyrosinase by different concentrations of salidroside

\subsection{Inhibitory Type of Salidroside on Tyrosinase}

The inhibitory type of mushroom tyrosinase of LDOPA by salidroside was also studied. The relationship between enzyme activity and different concentrations of salidroside was showed in Figure 2. The plots of the remaining enzyme activity versus enzyme concentrations at different concentrations of salidroside formed a series of straight lines which all passed through the origin. Increasing the concentration of inhibitor resulted in a descent of the line slope, indicating that the inhibition by salidroside on the activity of mushroom tyrosinase was reversible. So the effector reduced catalytic efficiency of enzyme by inhibiting enzyme activity, which leaded to the decrease of enzyme activity, not by reducing the amount of enzyme. Thus salidroside was a reversible inhibitor of mushroom tyrosinase for the oxidation of L-DOPA.

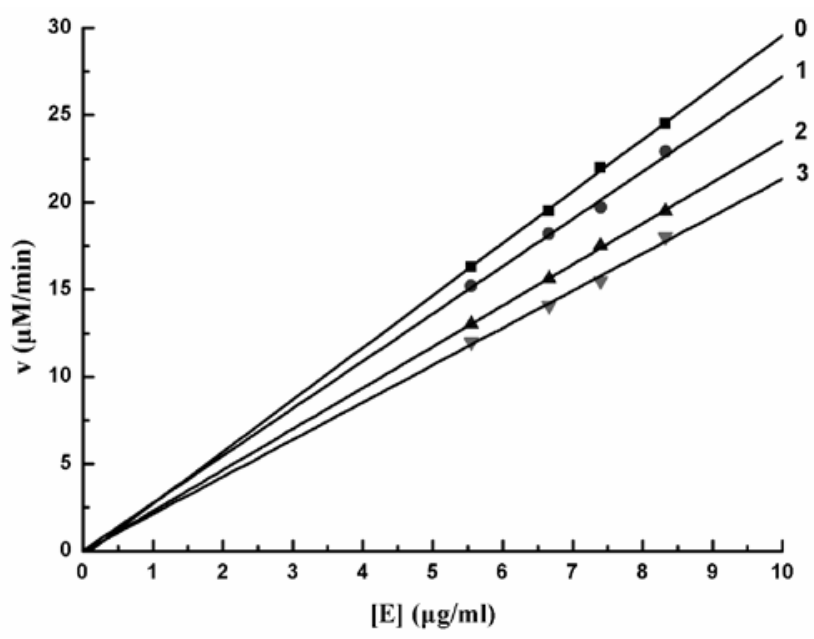

Figure 2. The catalytic activity of tyrosinase at different enzyme concentrations and different concentrations of salidroside. Concentrations of salidroside for curves 0 -3 were $0,0.1,0.25$, and 0.5 $\mathrm{mM}$, respectively

The kinetic behavior of mushroom tyrosinase during the oxidation of L-DOPA was investigated, and the oxidation of L-DOPA by mushroom tyrosinase followed Michaelis-Menten kinetics. Lineweaver-Burk plot yielded a series of straight lines intersecting at the ordinate (Figure 3). With increasing concentration of salidroside, $\mathrm{K}_{\mathrm{m}}$ increased, while $\mathrm{V}_{\max }$ was constant. Thus salidroside belonged to competitive inhibitor, and indicated that salidroside competed to the substrate with the free enzyme. The equilibrium constant for inhibitor binding with free enzyme, $\mathrm{K}_{\mathrm{I}}$, was obtained from a plot of the apparent Michaelis-Menten constant $\left(\mathrm{K}_{\mathrm{m}}{ }^{\mathrm{app}}\right)$ versus the concentration of salidroside, which was linear as shown in the inset. The obtained constant was $0.882 \mathrm{mM}$.

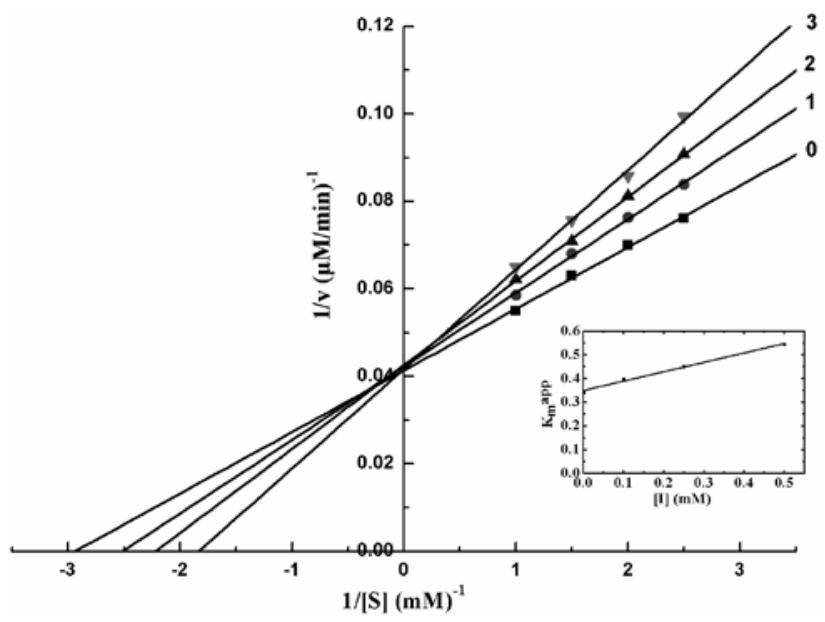

Figure 3. Lineweaver-Burk plots for the catalysis of L-DOPA by tyrosinase at $30^{\circ} \mathrm{C}, \mathrm{pH}$ 6.8. Concentration of salidroside for plots $0-3$ was $0,0.1,0.25$, and $0.5 \mathrm{mM}$, respectively. The inset represented the plot of apparent Michaelis constant $\left(\mathrm{K}_{\mathrm{m}}{ }^{\text {app }}\right)$ versus the concentration of salidroside for determining the inhibition constant $\mathrm{K}_{\mathrm{I}}$

\subsection{Analysis of UV Spectrum of Tyrosinase with Salidroside}

Tyrosinase including two copper ions located on helical bundle ( $\mathrm{CuA}$ and $\mathrm{CuB}$ ) in its active center belonged to the copper metalloprote. Particularly the active center had a high conservation, thus it played an important role in the catalytic process of tyrosinase [33]. In ultraviolet visible range of wavelengths, small molecule inhibitors with characteristic absorption would chelate with tyrosinase, which could enhance internal conjugation of inhibitors and ultraviolet absorption peak of inhibitors got redshifted [30,31].

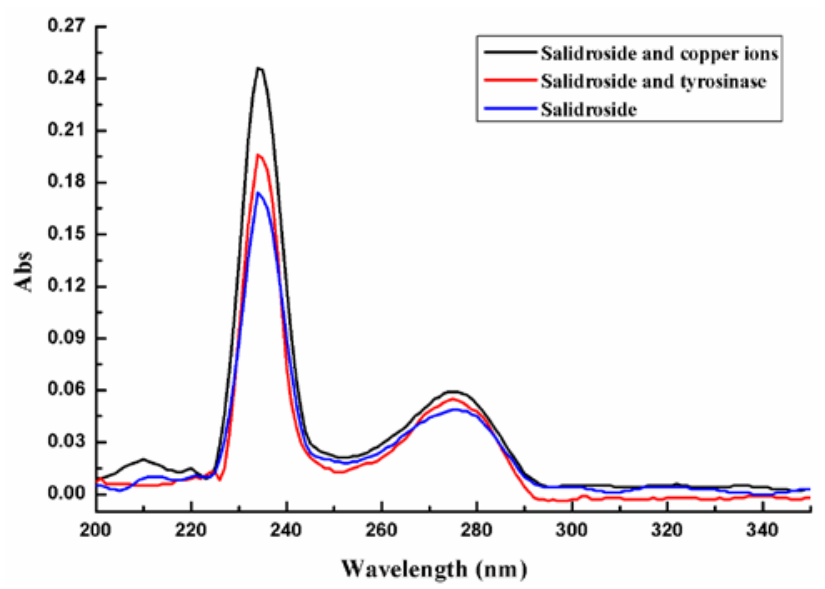

Figure 4. Ultraviolet-visible spectrum of each mixture in the wavelength of 200-600 nm. The three plots represented Ultraviolet-visible spectrum of each mixture only with salidroside, added tyrosinase and copper ions, respectively

The paper studied the ultraviolet absorption spectrum of salidroside when $\mathrm{CuSO}_{4}$ solution or tyrosinase was added. 
As Figure 4 shown, salidroside had the maximum absorption at $234 \mathrm{~nm}$ and $275 \mathrm{~nm}$. After putting excess copper ions into salidroside, the wavelength of characteristic absorption didn't change, which indicated that salidroside didn't chelate with copper ions. Similarly, when tyrosinase was added to the solution of salidroside, the wavelength of characteristic absorption still didn't change, which further illustrated that salidroside didn't chelate with copper ions in active center of tyrosinase.

Scanning the ultraviolet-visible spectroscopy in the reaction of $0,10,20,30$, and 40 min found that only when test tube was added to copper ions or sodium phosphate buffer, the ultraviolet absorption spectrum of salidroside didn't change over time. However, as Figure 5 shown, only when test tube was added to tyrosinase without substrate, salidroside could be catalyzed to produce reaction products which significantly enhanced the absorption in the area of ultraviolet and visible light, whereas, the product had significant absorption peak at $234 \mathrm{~nm}$ and $275 \mathrm{~nm}$, so it might be quinones. With the time going from 0 to $40 \mathrm{~min}$, the absorption of ultraviolet and visible light became stronger and stronger, and it meant that the reaction products were accumulated gradually. Salidroside had phenolic hydroxyl groups similar to tyrosine, thus perhaps enzymatic oxidation of hydroxyl group lead to the intensive absorption of light. Hence salidroside served as substrate analog of tyrosinase to react with tyrosine to product quinones which could enhance the light absorption [34].

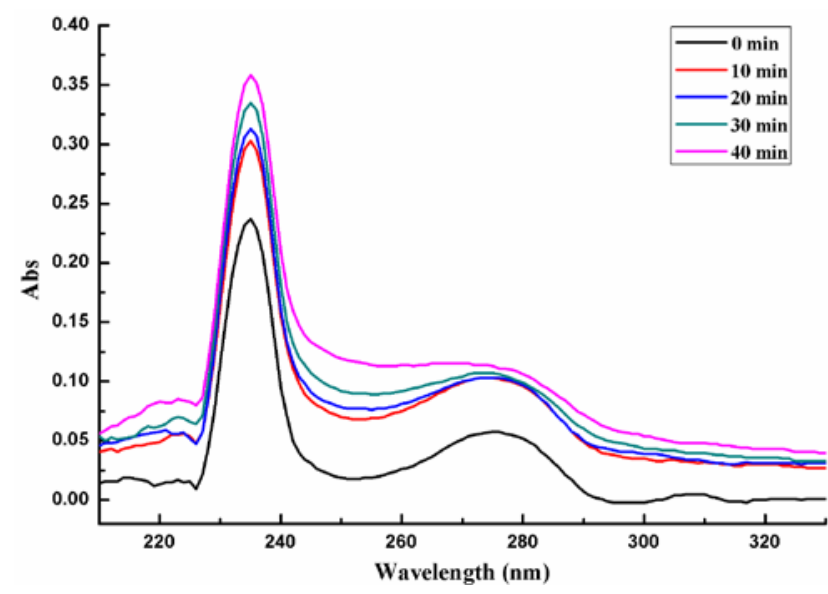

Figure 5. Ultraviolet-visible spectrum of each mixture of tyrosinase in the wavelength of 200-600 $\mathrm{nm}$ at different times. The five plots represented Ultraviolet-visible spectrum of each mixture in the reaction of $0,10,20,30$, and $40 \mathrm{~min}$, respectively

When seeking and developing a kind of anti-freckle product, not only should we consider that whether it had a mild and significant effect on skin or not, and at the same time if it was safe and reliable, but also we should consider its own economic and comprehensive benefits. Though some extracts in which salidroside was the main ingredient were low-purity, the methods of extraction and separation could be optimized to improve yield. The monomers related to plants such as salidroside could be artificially synthesized by the means of organic synthesis, which not only decreased complexity of manufacturing, but also reduced costs. Meanwhile some monomers related to plants had ability to anti-microbial, anti-lipid peroxidation, antihypertension and so on [24,25]. Hence the functions could be maximized by combining with modern biotechnology to achieve a kind of anti-freckle product with high comprehensive performance.

\subsection{Molecular Modeling for the Binding of Salidroside with Tyrosinase}

According to the result of modeling docking, salidroside was docked into the binding pocket of the tyrosinase. Detailed analysis showed that phenyl group fitted into the aromatic-rich binding pocket, surrounded by the aromatic side Phe59, Trp184, His54, His38 and His97 (Figure 6). Moreover, the phenyl ring of salidroside was vertical to the aromatic ring of residue Trp184, giving rise to a positive $\mathrm{CH}-\pi$ interaction. The glucose moeity of salidroside was located in the entrance of binding pocket of tyrosinase and made a high density of van del waals when it contacted with the around residues. Importantly, the 2-OH, 3-OH, 4-OH and 6-OH of glucose formed hydrogen bond interactions with residues His97, Asn191, Ala202 and Thr203, respectively, which was main binding affinity between salidroside and tyrosinase. All in all, docking results implied that the possible inhibitory mechanisms may be attributed to the weak interactions between salidroside and tyrosinase such as hydrogen bonds and van del waals.

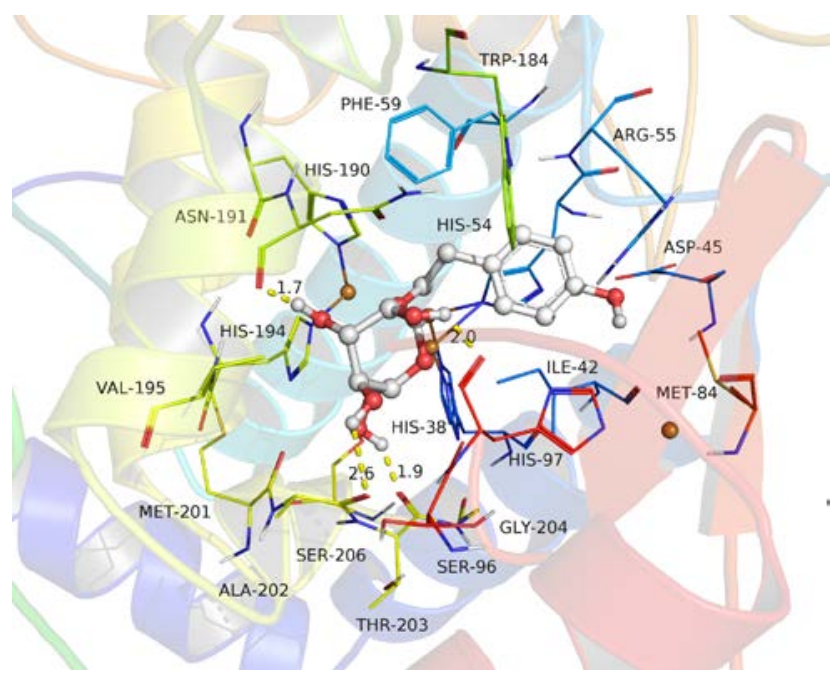

Figure 6. Detailed analysis of interactions between salidroside and tyrosinase

L-DOPA and salidroside had similar molecular structures, and according to the previous experimental results, it could be inferred that salidroside functioned as substrate analogs to react with tyrosinase. From the molecular structure point of view, L-DOPA was chelated with copper ions located in the active center of tyrosinase on account of the o-phenolic hydroxyl group which was in a plane, while salidroside was only with phenolic hydroxyl group, so as to salidroside couldn't chelate with copper ions. Furthermore, even if the glucose moeity of salidroside contained o-phenolic hydroxyl group, it also wasn't able to chelate with copper ions due to retrorse spatial structure and flexible tetrahydropyrane rings which were adverse to chelation. In a word, The results based on molecular docking further validated that salidroside didn't react with copper ions located in the active center of tyrosinase, which was in accordance with the conclusion achieved by UV spectrum. In addition, Donghyun Kim et al. had found that some flavonoids had a high inhibition to 
tyrosinase, which was related to the ability to form chelate with copper ions [35]. However, in this experiment, the inhibition of salidroside on tyrosinase was only up to $33.04 \%$. Thus, it could be speculated the reason that salidroside didn't have too high inhibition to tyrosinase was that the inhibitor couldn't react with copper ions located in the active center of tyrosinase. So far many researches had showed that flavonoids had a high inhibition to tyrosinase such as quercetin due to the similar structures to L-DOPA, and others such as rutin also had a great inhibition to tyrosinase which mainly reacted with copper ions located in the active center of enzyme [35,36]. In conclusion, it could be conjectured that the inhibition ability to inhibitors of tyrosinase was not only related to the similarity of substrates, but also associated with the extent of chelation between inhibitor and copper ions located in the active center of tyrosinase.

\section{Conclusions}

So far there had been some cosmetics added to extracts in which salidroside was the main ingredient, however, until now no researches had been carried to explore the inhibitory mechanism of salidroside on tyrosinase. Here, we found that salidroside showed certain inhibitory effects on enzyme activity by enzyme kinetics, moreover the highest inhibition rate was up to $33.04 \%$. Besides salidroside was also a competitive inhibitor of tyrosinase which served as a substrate analogue to combine with enzyme to produce quinones that could enhance the light absorption in ultraviolet-spectral analysis. Salidroside was a main kind of plant flavonoids from Rhodiola rosea, walnut and so on. Studying the inhibitory mechanism of salidroside on tyrosinase was not only helpful for developing anti-freckle products in which salidroside was one of the most important components, but also beneficial for making better use of plant flavonoids to seek for a new generation of natural skin care cosmetics. In addition, molecular docking showed that the weak interactions between salidroside and tyrosinase contained hydrogen bonds and van del waals, and it also gave us rational explanation of interaction between salidroside and tyrosinase, which provided valuable information for further study of tyrosinase inhibitors. Furthermore, according to the result of molecular docking, we conjectured that the inhibition ability to inhibitors of tyrosinase was associated with the similarity of substrates and the extent of chelation between inhibitor and copper ions located in the active center of tyrosinase. Thus, we could consider using molecular docking to optimize the molecular structure of salidroside or design structural analogues of salidroside so as to obtain some monomers with high inhibition rate of tyrosinase, which provided a new way to study the anti-freckle products.

\section{Acknowledgments}

The work was supported by the National Natural Science Foundation of China (Grant No. 21466037), Supporting Plan Issue of the Ministry of Science and Technology (2011BAD46B00) and the science and technology major program of Yunnan education bureau (ZD2014009).

\section{References}

[1] Ryu YB, W IM, Kang NS, Kim HY, Kim JH, Moon YH, Park KH: Kurarinol, tyrosinase inhibitor isolated from the root of Sophora flavescens. Phytomedicine 2008, 15(8): 612-618.

[2] Kang Y, Choi JU, Lee EA, Park HR: Flaniostatin, a new flavonoid glycoside isolated from the leaves of Cudrania tricuspidata as a tyrosinase inhibitor. Food Sci Biotechnol 2013, 22(5): 1449-1452.

[3] Yamaguchi Y, Brenner M, Hearing VJ: The regulation of skin pigmentation. J Biol Chem 2007, 282(38): 27557-27561.

[4] Eller MS, Ostrom K, Gilchrest BA: DNA damage enhances melanogenesis. P Natl Acad Sci USA 1996, 93(3): 1087-1092.

[5] Ortonne JP: Photoprotective properties of skin melanin. Brit $J$ Dermatol 2002, 146(s61): 7-10.

[6] Kim YJ, No JK, Lee JH, Chung HY: 4,4'-Dihydroxybiphenyl as a new potent tyrosinase inhibitor. Biol Pharm Bull 2005, 28(2): 323327.

[7] Gawande SS, Warangkar SC, Bandgar BP, Khobragade CN: Synthesis of new heterocyclic hybrids based on pyrazole and thiazolidinone scaffolds as potent inhibitors of tyrosinase. Bioorgan Med Chem 2013, 21(10): 2772-2777.

[8] Lou SN, Yu MW, Ho CT: Tyrosinase inhibitory components of immature calamondin peel. Food Chem 2012, 135(3): 1091-1096.

[9] Kim YJ, Uyama H: Tyrosinase inhibitors from natural and synthetic sources: structure, inhibition mechanism and perspective for the future. Cell Mol Life Sci 2005, 62(15): 1707-1723.

[10] Lin YF, Hu YH, Lin HT, Liu X, Chen YH, Zhang S, Chen QX: Inhibitory effects of propyl gallate on tyrosinase and its application in controlling pericarp browning of harvested longan fruits. J Agr Food Chem 2013, 61(11): 2889-2895.

[11] Chang TS: An updated review of tyrosinase inhibitors. Int $J$ Mol Sci 2009, 10(6): 2440-2475.

[12] Solano F, Briganti S, Picardo M, Ghanem G: Hypopigmenting agents: an updated review on biological, chemical and clinical aspects. Pigm Cell Res 2006, 19(6): 550-571.

[13] Kondo T, Hearing VJ: Update on the regulation of mammalian melanocyte function and skin pigmentation. Expert Rev Dermatol 2011, 6(1): 97-108.

[14] Lim YJ, Lee EH, Kang TH, Ha SK, Oh MS, Kim SM, Yooh TJ, Kang $\mathrm{CH}$, Park JH, Kim SY: Inhibitory effects of arbutin on melanin biosynthesis of a -melanocyte stimulating hormoneinduced hyperpigmentation in cultured brownish guinea pig skin tissues. Arch Pharm Res 2009, 32(3): 367-373.

[15] Cabanes J, Chazarra S, Garcia-Carmona F: Kojic Acid, a Cosmetic Skin Whitening Agent, is a Slow-binding Inhibitor of Catecholase Activity of Tyrosinase. J Pharm Pharmacol 1994, 46(12): 982-985.

[16] Briganti S, Camera E, Picardo M: Chemical and instrumental approaches to treat hyperpigmentation. Pigm Cell Res 2003, 16(3): 101-110.

[17] Kim KS, Kim JA, Eom SY, Lee SH, Min KR, Kim Y: Inhibitory effect of piperlonguminine on melanin production in melanoma B16 cell line by downregulation of tyrosinase expression. Pigm Cell Res 2006, 19(1): 90-98.

[18] Nakayama T, Sato T, Fukui Y, Yonekura-Sakakibara K, Hayashi H, Tanaka Y, Kusumi T, Nishino T: Specificity analysis and mechanism of aurone synthesis catalyzed by aureusidin synthase, a polyphenol oxidase homolog responsible for flower coloration. FEBS Letters 2001, 499(1): 107-111.

[19] Lin J, Chiang H, Lin Y, Wen K: Natural products with skinwhitening effects. J Food Drug Anal 2008, 16(2): 1-10.

[20] Peng LH, Liu S, Xu SY, Chen L, Shan YH, Wei W, Liang WQ, Gao JQ: Inhibitory effects of salidroside and paeonol on tyrosinase activity and melanin synthesis in mouse B16F10 melanoma cells and ultraviolet B-induced pigmentation in guinea pig skin. Phytomedicine 2013, 20(12): 1082-1087.

[21] Chen SF, Tsai HJ, Hung TH, Chen CC, Lee CY, Wu CH, Wang PY, Liao NC: Salidroside improves behavioral and histological outcomes and reduces apoptosis via PI3K/Akt signaling after experimental traumatic brain injury. Plos One 2012, 7(9): e45763. 
[22] Liu CY, Tai ZG, Feng SQ, Fang YS, Cai L, Ding ZT: Chemical constituents from the seed coat of Juglans regia. China. $J$ Chin Mater Med 2012, 37(10): 18.

[23] Calcabrini C, De Bellis R, Mancini U, Cucchiarini L, Potenza L, De Sanctis R, Patrone V, Scesa C, Dachà M: Rhodiola rosea ability to enrich cellular antioxidant defences of cultured human keratinocytes. Arch Dermatol Res 2010, 302(3): 191-200.

[24] Kang HS, Kim HR, Byun DS, Son BW, Nam TJ, Choi JS: Tyrosinase inhibitors isolated from the edible brown algaEcklonia stolonifera. Arch Pharm Res 2004, 27(12): 1226-1232.

[25] Kucinskaite A, Briedis V, Savickas A: Experimental analysis of therapeutic properties of Rhodiola rosea $L$. and its possible application in medicine. Med 2003, 40(7): 614-619.

[26] Zhong H, Xin H, Wu LX, Zhu YZ: Salidroside attenuates apoptosis in ischemic cardiomyocytes: a mechanism through a mitochondria-dependent pathway. J Pharmacol Sci 2009, 114(4): 399-408.

[27] Momtaz S, Lall N, Basson A: Inhibitory activities of mushroom tyrosine and DOPA oxidation by plant extracts. S Afr J Bot 2008, 74(4): 577-582.

[28] Mu Y, Li L, Hu SQ: Molecular inhibitory mechanism of tricin on tyrosinase. Spectrochim Acta A 2003, 107(15): 235-240.

[29] Xie LP, Chen QX, Huang H, Wang HZ, Zhang RQ: Inhibitory effects of some flavonoids on the activity of mushroom tyrosinase. Biochemistry (Moscow) 2003, 68(4): 487-491.
[30] Kubo I, Kinst-Hori I: Flavonols from saffron flower: tyrosinase inhibitory activity and inhibition mechanism. J Agr Food Chem 1999, 47(10): 4121-4125.

[31] Kim D, Park J, Kim J, Han C, Yoon J, Kim N, Seo J, Lee C: Flavonoids as mushroom tyrosinase inhibitors: a fluorescence quenching study. J Agr Food Chem 2006, 54(3): 935-941.

[32] Trott O, Olson AJ: AutoDock Vina: improving the speed and accuracy of docking with a new scoring function, efficient optimization, and multithreading. J Comput Chem 2010, 31(2): 455-461.

[33] Matoba Y, Kumagai T, Yamamoto A, Yoshitsu H, Sugiyama M: Crystallographic evidence that the dinuclear copper center of tyrosinase is flexible during catalysis. $J$ Biol Chem 2006, 281(13): 8981-8990.

[34] Kubo I, Nihei K, Tsujimoto K: Methy p-coumarate, a melanin formation inhibitor in B16 mouse melanoma cells. Bioorg Med Chem 2004, 12(20): 5349-5354.

[35] Kim D, Park J, Kim J, Han C, Yoon J, Kim N, Seo J, Lee C: Flavonoids as mushroom tyrosinase inhibitors: a fluorescence quenching study. J Agr Food Chem 2006, 54(3): 935-941.

[36] Si YX, Yin SJ, Oh S, Wang ZJ, Ye S, Yan L, Yang JM, Park YD, Lee J, Qian GY: An integrated study of tyrosinase inhibition by rutin: progress using a computational simulation. J Biomol Struct Dyn 2012, 29(5): 999-1012. 